Gut and Liver, Vol. 11, No. 2, March 2017, pp. 169-170

EDITORIAL

\title{
Do We Need to Retest of Helicobacter pylori Infection after Gastric Cancer Surgery?
}

\author{
Sang Kil Lee
}

Division of Gastroenterology, Department of Internal Medicine, Severance Hospital, Institute of Gastroenterology, Yonsei University College of Medicine, Seoul, Korea

See "Dynamic Changes in Helicobacter pylori Status Following Gastric Cancer Surgery" by Kichul Yoon, et al. on page 209, Vol. 11. No. 2, 2017

Helicobacter pylori is a human pathogen and can lead to chronic gastritis, peptic ulcer disease, gastric adenocarcinoma and mucosa-associated lymphoid tissue lymphoma. $H$. pylori infection is associated with gastric cancer and has been categorized as a group I carcinogen in gastric cancer. In 2016, Maastricht V/Florence Consensus report emphasized $H$. pylori infection as an infectious disease and reinforced the statement that $H$. pylori eradication can cure gastritis and alter the progression to long-term complications, or recurrence of disease. ${ }^{1,2}$ Thus, screening and eradication for $H$. pylori is recommended in individuals at increased risk for gastric cancer.

Although $H$. pylori eradication after gastric cancer surgery is also recommended, ${ }^{1,3}$ it was not clear that eradication therapy could reduce the risk of metachronous gastric cancer in the remnant stomach after partial gastrectomy. A recent prospective randomized clinical trial in Korea reported that gastric cancer recurrence rate was not different according to $H$. pylori eradication treatment (5-year gastric cancer recurrence rates, 4.6\% in the treatment group vs $8.5 \%$ in the placebo group; $p=0.652) .{ }^{4}$ In this kind of study, it is necessary to be able to control or eliminate influences by the timing of eradication, intragastric environmental change due to gastrectomy and spontaneous regression of $H$. pylori infection in order to confirm the effectiveness of the eradication treatment.

There are a several things to consider when deciding to treat. The first is the time of eradication. Postoperative eradication therapy is preferred to preoperative eradication therapy because of several reasons in clinics. Additional 1 or 2 weeks is needed for the eradication therapy before surgery. Adverse events as- sociated with antibiotics may occur and delay the surgery. In cases with unplanned total gastrectomy, eradication therapy would not be unnecessary. The second is that even if eradication was not tried, H. pylori can spontaneously disappear. ${ }^{3}$ In general, $H$. pylori infection persists if not eradicated, whereas the spontaneous regression is happened in the remnant stomach. The overall spontaneous clearance rate of $H$. pylori infection after partial gastrectomy was reported $38.6 \%$ to $43 \%$. ${ }^{4,5}$ Previous study reported that the spontaneous clearance rate of H. pylori was related to operative procedures. ${ }^{6}$ The Billroth-II procedure had a higher bile reflux rate and a lower $H$. pylori infection prevalence than the Billroth-I procedure. Bile reflux is a pathogenic factor in gastric remnant reflux gastritis and induces chronic histological inflammation. ${ }^{7}$ Third, if you decide to treat, we have to decide to re-test postoperative status of $H$. pylori. It was assumed that the change of $\mathrm{pH}$ and bile reflux in the remnant stomach might affect $H$. pylori colonization. ${ }^{5,8,9}$ Hypochlorhydric environment caused by antrectomy and increased bile reflux due to the loss of pyloric ring might inhibit the growth of $H$. pylori. And rate of $H$. pylori infection decreased over time after partial gastrectomy. A study in Taiwan reported the prevalence of $H$. pylori 1 to 15 years after surgery was $29.5 \%$, after 16 to 30 years it was $13.6 \%$, and after more than 30 years it was $10 \%{ }^{9}$ However, studies on how $H$. pylori infections change after gastrectomy are still lacking.

The article published in Gut and Liver, "Dynamic changes in Helicobacter pylori status following gastric cancer surgery" by Yoon et al. ${ }^{10}$ started from a question for clinicians about the need for retest postoperative $H$. pylori status. In this study, they

Correspondence to: Sang Kil Lee

Division of Gastroenterology, Department of Internal Medicine, Severance Hospital, Institute of Gastroenterology, Yonsei University College of

Medicine, 50-1 Yonsei-ro, Seodaemun-gu, Seoul 03722, Korea

Tel: +82-2-2228-1996, Fax: +82-2-393-6884, E-mail: sklee@yuhs.ac

pISSN 1976-2283 eISSN 2005-1212 https://doi.org/10.5009/gnl16608

(a) This is an Open Access article distributed under the terms of the Creative Commons Attribution Non-Commercial License (http://creativecommons.org/licenses/by-nc/4.0) which permits unrestricted non-commercial use, distribution, and reproduction in any medium, provided the original work is properly cited. 
evaluated the postoperative changes of $H$. pylori detection and analyzed the factors which affect changes of in $H$. pylori infection status after gastric cancer surgery. One hundred forty patients who underwent curative gastrectomy and had a positive $H$. pylori status without eradication therapy at the time of gastric cancer diagnosis were prospectively enrolled and annual followup was done to evaluate postoperative $H$. pylori status. The rate of spontaneous negative conversion at least once during followup was 42.9\% (60/140). And of these 60 patients, eight (13.3\%) showed more complex postoperative dynamic changes between negative and positive results after being confirmed as spontaneous negative conversion. The author analyzed factors associated with $H$. pylori status and demonstrated that the spontaneous negative conversion group showed a trend of more postoperative intestinal metaplasia compared to the persistent $H$. pylori group (36.7\% [22/60] vs $16.3 \%$ [13/80], $\mathrm{p}=0.001)$. Unlike other previous study, ${ }^{11}$ there was no significant difference between spontaneous conversion and persistently positive groups according to the surgery type which could affect the bile reflux. The peculiarities of this study were that the intestinal metaplasia of the remnant stomach had a greater effect on the presence of $H$. pylori than bile reflux which can be affected by types of surgery. The author demonstrated that these results could suggest that intestinal metaplasia could be a more important factor for the survival of $H$. pylori than bile reflux. However, this finding needs to be confirmed by further studies.

The present study showed that there was relatively high percentage of spontaneous regression and dynamic changes in status of $H$. pylori after partial gastrectomy, in case of more histologic intestinal metaplasia in the remnant stomach in postoperative period. Inaccurate diagnosis for status of $H$. pylori may lead to unnecessary eradication therapy and underestimate the protective effect of eradication therapy in patients with gastric cancer surgery. This clinical evidence could support a need to retest of postoperative $H$. pylori status before eradication therapy. However, it still cannot give answer as to how and when to test $H$. pylori after gastrectomy, more research is needed.

\section{CONFLICTS OF INTEREST}

No potential conflict of interest relevant to this article was reported.

\section{REFERENCES}

1. Malfertheiner P, Megraud F, O'Morain CA, et al. Management of Helicobacter pylori infection-the Maastricht V/Florence Consensus Report. Gut 2017;66:6-30.

2. Lee YC, Chiang TH, Liou JM, Chen HH, Wu MS, Graham DY. Mass eradication of Helicobacter pylorito prevent gastric cancer: theoretical and practical considerations. Gut Liver 2016;10:12-26.

3. Fock KM, Katelaris P, Sugano K, et al. Second Asia-Pacific Consensus guidelines for Helicobacter pylori infection. J Gastroenterol Hepatol 2009;24:1587-1600.

4. Kim YI, Cho SJ, Lee JY, et al. Effect of Helicobacter pylori eradication on long-term survival after distal gastrectomy for gastric cancer. Cancer Res Treat 2016;48:1020-1029.

5. Bair MJ, Wu MS, Chang WH, et al. Spontaneous clearance of Helicobacter pylori colonization in patients with partial gastrectomy: correlates with operative procedures and duration after operation. J Formos Med Assoc 2009;108:13-19.

6. Suh S, Nah JC, Uhm MS, et al. Changes in prevalence of Helicobacter pylori infection after subtotal gastrectomy. Hepatogastroenterology 2012;59:646-648.

7. Lin YS, Chen MJ, Shih SC, Bair MJ, Fang CJ, Wang HY. Management of Helicobacter pylori infection after gastric surgery. World J Gastroenterol 2014;20:5274-5282.

8. Kato T, Motoyama H, Akiyama N. Helicobacter pylori infection in gastric remnant cancer after gastrectomy. Nihon Rinsho 2003;61:30-35.

9. Abe H, Murakami K, Satoh S, et al. Influence of bile reflux and Helicobacter pylori infection on gastritis in the remnant gastric mucosa after distal gastrectomy. J Gastroenterol 2005;40:563-569.

10. Yoon K, Kim N, Kim J, et al. Dynamic changes in Helicobacter pylori status following gastric cancer surgery. Gut Liver 2017;11:209-215.

11. Cho SJ, Choi IJ, Kook MC, et al. Randomised clinical trial: the effects of Helicobacter pylori eradication on glandular atrophy and intestinal metaplasia after subtotal gastrectomy for gastric cancer. Aliment Pharmacol Ther 2013;38:477-489. 